\title{
Synthesis and the Effects of New Melamine Superplasticizer on the Properties of Concrete
}

\author{
Huqun Wang, ${ }^{1}$ Xiaofeng Yang, ${ }^{1}$ Weifeng Xiong, ${ }^{1}$ Xiaobin Liu, ${ }^{1}$ and Zhimin Zhang ${ }^{2}$ \\ ${ }^{1}$ Technology Center, Beijing Oriental Yu Hong Waterproof Technology Co. Ltd., Beijing 101309, China \\ ${ }^{2}$ School of Chemistry and Chemical Engineering, Shanxi University, Taiyuan 030006, China \\ Correspondence should be addressed to Huqun Wang; wanghuqun@yahoo.cn
}

Received 13 June 2013; Accepted 7 July 2013

Academic Editors: T. García, C.-T. Hsieh, and Y. Otsubo

Copyright (c) 2013 Huqun Wang et al. This is an open access article distributed under the Creative Commons Attribution License, which permits unrestricted use, distribution, and reproduction in any medium, provided the original work is properly cited.

\begin{abstract}
This work deals with the synthesis of new melamine superplasticizer (NMS) with high molecule weight and comparatively high processing thermostability by the reaction among melamine, formaldehyde, and sulfonated glucose. The surface activity and the structure of new melamine superplasticizer and their performance in concrete were evaluated. The effect of molecular weight of synthesized new melamine superplasticizer on the performance of the concrete was determined. The results showed that the new melamine superplasticizer has been proved to be more efficient as a superplasticizer used in concrete.
\end{abstract}

\section{Introduction}

Superplasticizers (SPs) are recognized as important admixtures for their applications in modern concrete technology. Efforts have long been made by scientists and technologists to keep fresh concrete fluid to provide homogeneous transport of all particles in the building industry [1]. Traditional techniques which use the superplasticizers such as sulfonated melamine formaldehyde condensate (SMF) and sulfonated naphthalene formaldehyde condensate play a positive role in maintaining high fluiding concrete. The superplasticizers interact with the surface of cement particles and involve dispersion and decreasing of the natural tendency to coagulate in concrete. Polycarboxylates (PC) are recently commonly used as superplasticizers to disperse cement particles in concrete and mortar. However, it has been known that the dispersing force of PC for cement and its time variation are significantly affected by even slight differences in concrete components or mixing conditions. Such instability of the dispersing force is explained by the incompatibility between cement and PC [2-4]. Moreover, the side chain of the copolymer-type PC is composed of polyethylene oxide units. The methyl oxide bonds are easy to break under heat and oxygen. Furthermore, as a new generation water-reducing agent, the PC waterreducing agent would face the problem of short resources in the near future for nonregenerate ability of crylic acid derived from petroleum, so melamine superplasticizer has shown great advantage. Melamine superplasticizer is one of the most commonly employed due to its colorless, nontoxic, and good thermal stability [5-8]. However, the application of traditional melamine water reducing agent is becoming less and less due to the complex production process, high cost, and relatively inferior slump loss. Along with the architecture industrial development and on the concrete performance requirements to improve, the development of high performance superplasticizer is imperative.

In this study, a new melamine superplasticizer (NMS) was synthesized through polymerization of sulfonated melamine formaldehyde using sulfonated glucose as a new monomer. The chemical structure of NMS was characterized by FTIR spectra. The NMS was used as the superplasticizer in cement and concrete formation and showed high water reduction rate and retardation effect of slump loss based on its dosage.

\section{Experimental}

2.1. Materials. The cement used was normal number 425 Portland cement manufactured by Tangshan Cement Plant; the China ISO standard sand (standard sand for strength determination of cement to ISO679 and EN 196-1) was used. 
TABLE 1: Characteristics of cement.

\begin{tabular}{lcccccccc}
\hline Characteristic & $\mathrm{CaO}$ & $\mathrm{SiO}_{2}$ & $\mathrm{Al}_{2} \mathrm{O}_{3}$ & $\mathrm{Fe}_{2} \mathrm{O}_{3}$ & $\mathrm{MgO}$ & $\mathrm{SO}_{3}$ & $\mathrm{Na}_{2} \mathrm{O}$ & Loss \\
Mass\% & 57.64 & 25.32 & 6.62 & 2.07 & 1.73 & - & 0.31 & 6.21 \\
\hline
\end{tabular}

The characteristics of the cement are shown in Table 1. The results analyzed of the cement were as follows (mass \%): $\mathrm{C}_{3} \mathrm{~S} 53.6 \%, \mathrm{C}_{2} \mathrm{~S} 21.6 \%, \mathrm{C}_{3} \mathrm{Al} 10.9 \%, \mathrm{C}_{4} \mathrm{AF} 7.5 \%$, free $\mathrm{CaO}$ $1.7 \%, \mathrm{CaSO}_{4} \cdot 1 / 2 \mathrm{H}_{2} \mathrm{O} 2.2 \%, \mathrm{CaSO}_{4} 2.4 \%$, and $\mathrm{CaSO}_{4} \cdot 2 \mathrm{H}_{2} \mathrm{O}$ $0 \%$. The specific surface area (FBT-9 Tester specific surface area instrument) was found to be $3312 \mathrm{~cm}^{2} / \mathrm{g}$, and the particle size analysis using a Helos-Sucell laser particle size analyzer (SYMPATEC Instruments, Germany) shows that the volume average mean particle size is about $25 \mu \mathrm{m}$, and the median diameter $\mathrm{d} 50$ is about $20 \mu \mathrm{m}$. Specific density of this cement was about $3.12 \mathrm{~kg} / \mathrm{cm}^{3}$. The sand diameter is between $20-$ 40 mesh number, and cobble stone diameter is between 5$20 \mathrm{~mm}$. SMF is the commercial sample in China market.

2.2. Synthesis of NMS Resin. All reagents were of analytical reagent grade and were used without any further purification. The glucose and methylene chloride are added separately to the flasks and heated up to $60^{\circ} \mathrm{C}$. Thus, a certain amount of chlorosulfonic acid was dropped to the solution which was reacted for $4 \mathrm{~h}$. A little water and a small amount of alcohol were added and white precipitate was obtained by washing filtering, and the yield was $87 \%$. The precipitation is not needed to purify for the next use, and the framework was analyzed by ${ }^{1} \mathrm{HNMR}$. Because the precipitation is admixture, the peak may be attributed to the peaks of products. ${ }^{1} \mathrm{HNMR}$ data: (300 MHz, DMSO, ppm) $\delta: 9.586(1 \mathrm{H}, \mathrm{s}), 4.272-2.806$ $(3 \mathrm{H}, \mathrm{s}), 2.606(1 \mathrm{H}, \mathrm{s})$.

A water solution of melamine was mixed with sulfonated glucose as monomers, and a formaldehyde solution of distilled water was added to the previously mentioned solution under vigorous stirring at $65^{\circ} \mathrm{C}$. The $\mathrm{pH}$ of the solution was adjusted to about 11 by $30 \% \mathrm{NaOH}$ solution. After $1 \mathrm{~h}$, the middle production so obtained had been left by the addition sulfonating reagent $\left(\mathrm{NaHSO}_{3}\right)$ at $85^{\circ} \mathrm{C}$ temperature to ensure the completion of the substituted reaction. Subsequently the catalysts were added to the solution, and the polymerization reaction was processed for $2 \mathrm{~h}$. Finally, the $\mathrm{pH}$ was adjusted to about 7-8. The yellow viscous liquid was obtained, and the special spray drying method was used to obtain white free flowing powder melamine superplasticizer. The reaction process is seen in Scheme 1.

2.3. Materials Measurements. The IR spectra of NMS were recorded with an FT-IR spectrometer (Bruker Tensor 27). For the measurement of the thermal analysis of water-reducing agents on the surface of NMS and molecular weight of NMS, the TGA technology (MK II, UK) and GPC (Waters $1525 / 2414$, USA) were used, respectively.

2.4. Adsorption Amount Measurement and $\zeta$-Potential Measurement. A series of NMS solutions with different concentrations were prepared. Then, cement was added with a weight ratio of solid to solvent $1: 0.8$. After being stirred for $10 \mathrm{~min}$, the mixture was separated by centrifugation. The concentrations of NMS in the aqueous phase were measured by UV adsorption spectrophotometer (TU-1900, Beijing). The quantities adsorbed were calculated from the differences in the concentration of water-reducing agent in the liquid phase before and after adsorption.

For $\zeta$-potential measurements, the DBL-B microelectrophoresis analyzer was used. A certain amount of cement was added to the solution with a certain concentration of water-reducing agent. The ratio of solute to solvent by weight was $0.0025: 1$. After mixing for $5 \mathrm{~min}$ in the blender, the $\zeta$ potential of particles in the clarified liquor was analyzed, and the average of ten per measurement was regarded as the $\zeta$ potential of the cement particles.

2.5. The Application of Concrete. A certain amount of waterreducing agent was added to water in advance. Then Concrete was prepared with a cement/sand/cobblestone weight ratio of $1: 2.19: 3.73$. The fluidity of concrete was evaluated by the pullout spread of a sample from a flow cone specified according to GB8076-2008. The maximum diameter of the spread sample and the maximum width perpendicular to that diameter were measured, and the average of these two values was defined as the flow value. At the ages of 28 days, the compressive strength test was conducted on six pieces of prisms according to the Chinese standard GB/T176711999. The notched Charpy flexural strength of every mixture was measured on three prismatic specimens with ZBC-4B impact testing machine from Xinsansi Co. (Shenzhen, China) according to ISO179-1993 (E).

\section{Results and Discussion}

3.1. Thermal Analysis. Thermal stability of NMS whose TGA diagrams were shown in Figure 1 plays an important role in their applications. There are two main stages. Weight loss in the range of $200-240^{\circ} \mathrm{C}$ was about $20 \%$ mass losses, mainly due to the decomposition of the small molecules of short chain; weight loss in the range of $290-360^{\circ} \mathrm{C}$ was about $80 \%$ mass losses due to the formation of the higher thermal stability of cross-linked polymer yielded by use of sulfonated glucose. Weight loss temperature $(5 \mathrm{wt} \%$ weight loss percentages) of the sulfonated glucose samples is about $294^{\circ} \mathrm{C}$, and that of NMS is $308^{\circ} \mathrm{C}$, which indicates that the prepared particles have good thermal stability. In Figure 1, the TGA analysis of SMF is identical when comparing with NMS, but the decomposing temperature of SMF is lower than the NMS. The main reason is that the triazine ring of melamine enhances the rigidity of the chain and improves the energy of molecular thermal motion. By introducing small amounts of sulfonated glucose to the melamine molecular, the concentration of ethylene bond dropped and the molecular chain flexibility declined, which cause the increase of decomposition temperature [9].

3.2. Adsorption Property and the Effect of Various WeightAverage MW Resins for Adsorption Amount Changes. 


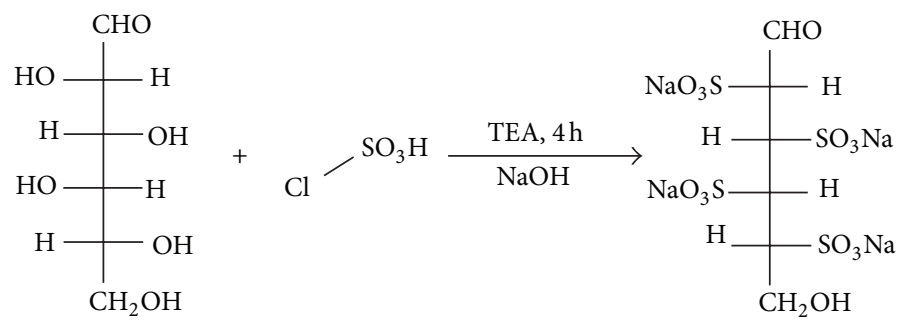<smiles>Nc1nc(N)nc(N)n1</smiles>

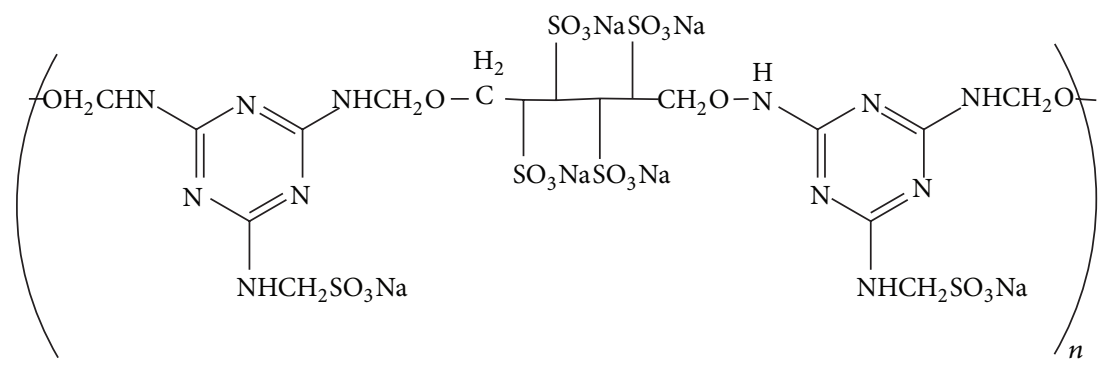

Scheme 1: Synthesis of new melamine superplasticizer (NMS).

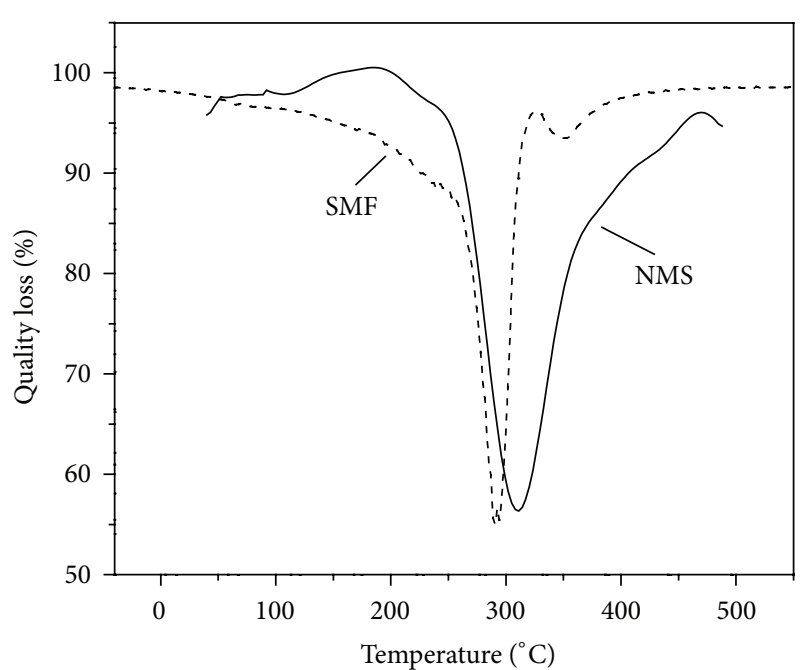

FIGURE 1: The TGA curve of new melamine superplasticizer (NMS) and SMF.

Figure 2 showed the reciprocal values of concentration of water-reducing agent and adsorption amount. The concentration change amounts to $10 \mathrm{~g} / \mathrm{L}$ after NMS adsorption for $10 \mathrm{~min}$. The semilogarithmic plots of concentration data gave a curve. The correlation constant for the fitted line was calculated to be $r=0.9905$ and $r=0.9964$, respectively, for SMF and NMS. It can be concluded that the Langmuir single layer adsorption occurred during the process of adsorption of NMS on cement particles. As shown in Figure 2, the adsorption of superplasticizer on gypsum surface approximately conformed to Langmuir's adsorption isotherm equation is as follows:

$$
\tau=\tau_{\infty} \frac{k c}{1+k c}
$$

where $\tau$ is the adsorbed amount, in $\mathrm{mg} / \mathrm{g} ; \tau_{\infty}$ is the saturated adsorbed amount, in $\mathrm{mg} / \mathrm{g}$; $c$ is the superplasticizer concentration, in $\mathrm{g} / \mathrm{L}$; and $k$ is the adsorption constant. The previous formula can be converted into the following:

$$
\frac{1}{\tau}=\frac{1}{\tau_{\infty}}+\frac{1}{\tau_{\infty} k c} .
$$

The $\tau_{\infty}$ can be assessed through (2) by the linear relationship of $1 / \tau$ versus $1 / c$ and then calculating the cut length at the $1 / \tau$ axis. This finding indicated that the single layer adsorption of NMS could be described by the Langmuir equation which was applied to quantify adsorption capacity, which gives the values of the maximum adsorption capacities of SMF and NMS 11.69 and $13.15 \mathrm{mg} / \mathrm{g}$, respectively, according to the intercept and slope of these lines, respectively [10].

Figure 3 shows the adsorption amounts of various weightaverage MW NMS resins on the surface of cement particles, when the amount of dispersants added increases from 2 to $10 \mathrm{~g} / \mathrm{L}$, respectively. The adsorption amount of the dispersant 


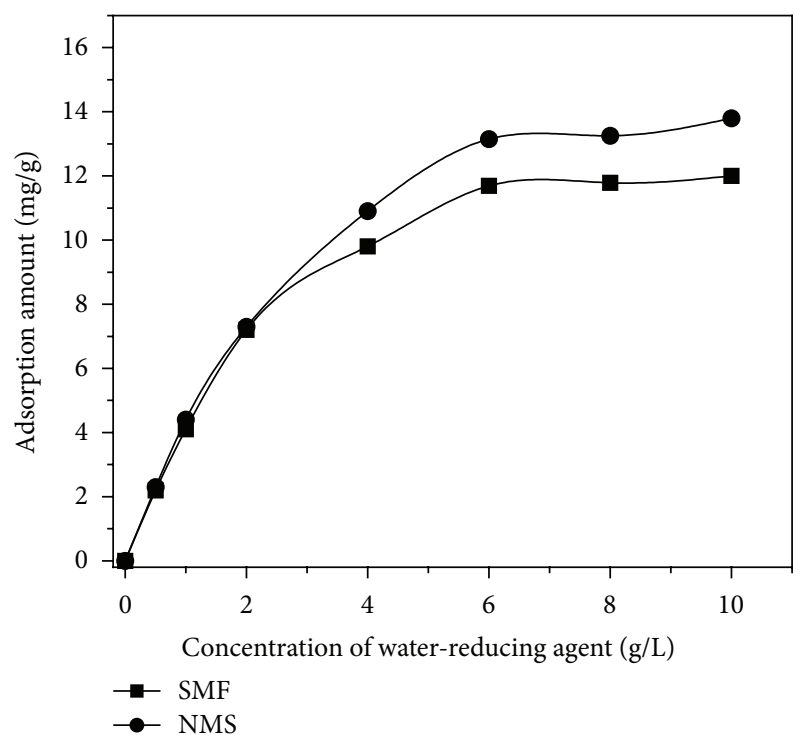

FIGURE 2: The curve of concentration and adsorption amount of water-reducing agent $\left(T=20^{\circ} \mathrm{C}\right)$.

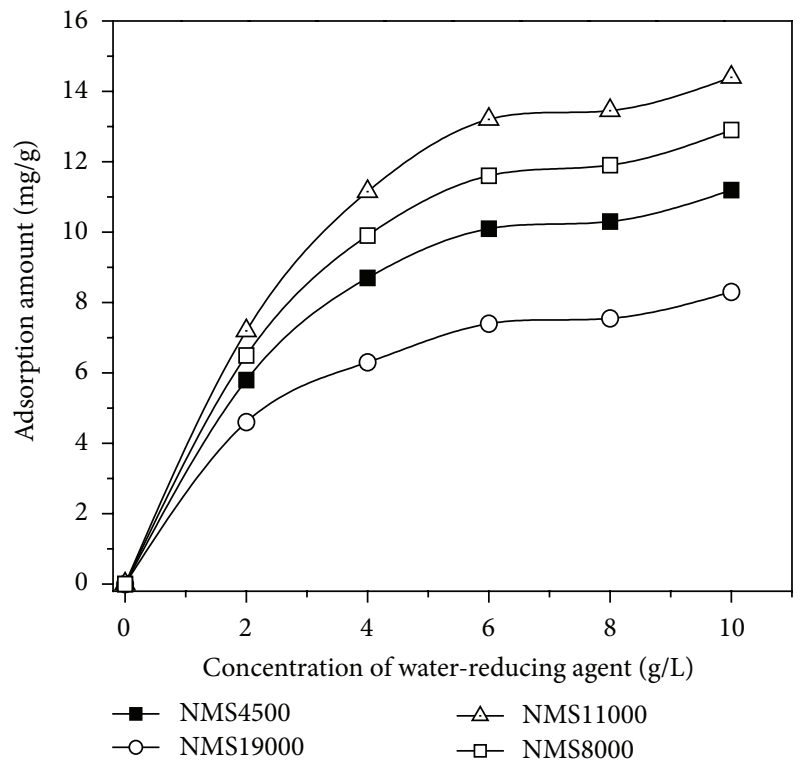

FIGURE 3: Adsorption amount changes of various weight-averages MW with water-reducing agent $\left(T=20^{\circ} \mathrm{C}\right)$.

onto the cement particles is generally determined by the functional groups in its molecule and MW. Generally, the adsorption amount increases with the increase of MW in an adequate time. Figure 3 shows that the resin with 11000 weight-average MW has a higher adsorption amount than those with over 11000 weight-averages MW. This is because bigger molecules have lower moving speed, and it is difficult for them to attain equilibrium in a short time. With the increase of molecular weight, the content of sulfonic acid groups of single molecules also increases, and large molecules containing more sulfonic acid groups are conducive to the increase in the adsorption at the surface of solid particles;

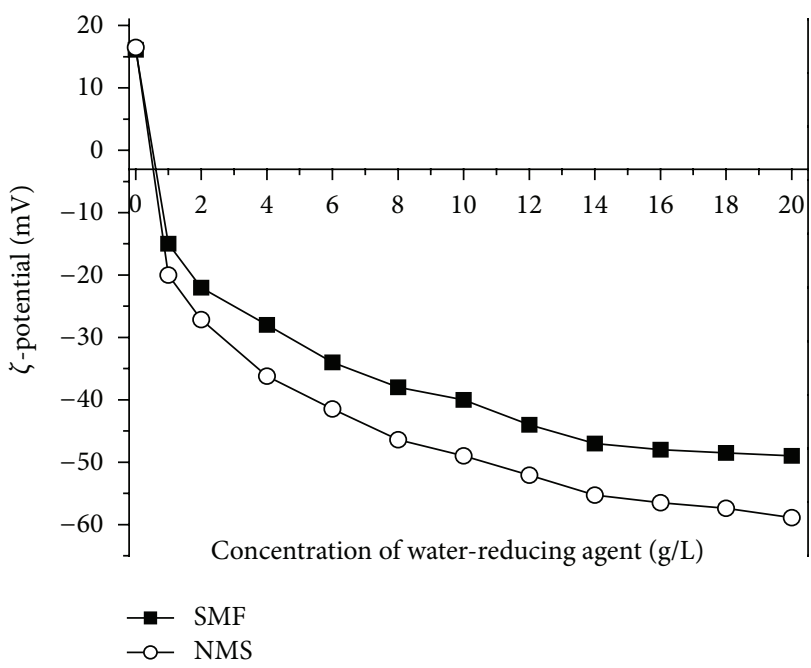

FIGURE 4: $\zeta$-potential of water-reducing agents with different concentrations.

thus, the adsorption is more firm. Sulfonic acid groups of lower density are conducive to the formation of curling configuration in solution, and greater electrostatic repulsion is produced by the adsorption on the solid particle surface.

3.3. Effect of Adsorption of Water-Reducing Agents on $\zeta$ Potential of Solid-Liquid Interface. As shown in Figure 4, $\zeta$ potential of cement particle with SMF and NMS changed from positive $(17 \mathrm{mV})$ to negative $(-25.34$ and $-57.01 \mathrm{mV})$ with the concentration of anionic surfactants increasing from 0 to $20 \mathrm{~g} / \mathrm{L}$. The reason for that was the adsorption of SMF, and NMS changed the charge distribution of the electrical double layer of the cement surface [11]. Figure 4 further shows that $\zeta$-potential of cement particle with NMS was higher than that of SMF at the same concentration. There were more sulfuric acid groups in NMS, which indicated that the electrostatic repulsion force between cement particles with NMS was higher.

3.4. Structure Analysis of the NMS. As shown in Figure 5, IR spectrum of NMS (a) had two new and strong characteristic absorption peaks in comparison with the IR spectrum of SMF (a). The peaks located at $1149 \mathrm{~cm}^{-1}$ and $1296 \mathrm{~cm}^{-1}$ were asymmetric and symmetric stretching vibration of $\mathrm{S}-$ $\mathrm{O}$ stretching vibration characteristic absorption peak and $\mathrm{S}=\mathrm{O}$ symmetrical stretching vibration, respectively, which indicated that a lot of sulfuric acid groups were introduced into the NMS molecule [12]. The peak located at $678 \mathrm{~cm}^{-1}$ belongs to the $\mathrm{C}-\mathrm{S}$ stretching vibration absorption peak, indicating that the copolymer sulfonic acid group contained, which was obviously stronger vibration than SMF. So, the sulfonated glucose has been embedded in the synthesized compound molecular structure.

3.5. Water Reduction and Slump Loss in Concrete. The evaluation of slump loss is important for field application. A lower degree of slump loss will prolong the time available for 
TABLE 2: Properties of superplasticized concrete with NMS.

\begin{tabular}{|c|c|c|c|c|c|}
\hline $\begin{array}{l}\text { Concrete } \\
\text { type }\end{array}$ & $\begin{array}{c}\text { Water-reducing rate } \\
(\%)\end{array}$ & $\begin{array}{l}\text { Initial slump flow } \\
(\mathrm{mm})\end{array}$ & $\begin{array}{c}60 \text { min slump flow } \\
(\mathrm{mm})\end{array}$ & $\begin{array}{c}\text { Water content } \\
\left(\mathrm{kg} / \mathrm{m}^{3}\right)\end{array}$ & $\begin{array}{c}\text { Air content } \\
(\%)\end{array}$ \\
\hline $\mathrm{CC}$ & Standard & 205 & 30 & 2.2 & 3.2 \\
\hline SMF 1.0 & 18.8 & 200 & 95 & 1.8 & 3.0 \\
\hline NMS 1.0 & 22.7 & 210 & 205 & 1.7 & 3.1 \\
\hline NMS 1.5 & 27.3 & 200 & 215 & 1.6 & 2.6 \\
\hline NMS 2.0 & 29.5 & 195 & 220 & 1.55 & 2.2 \\
\hline
\end{tabular}

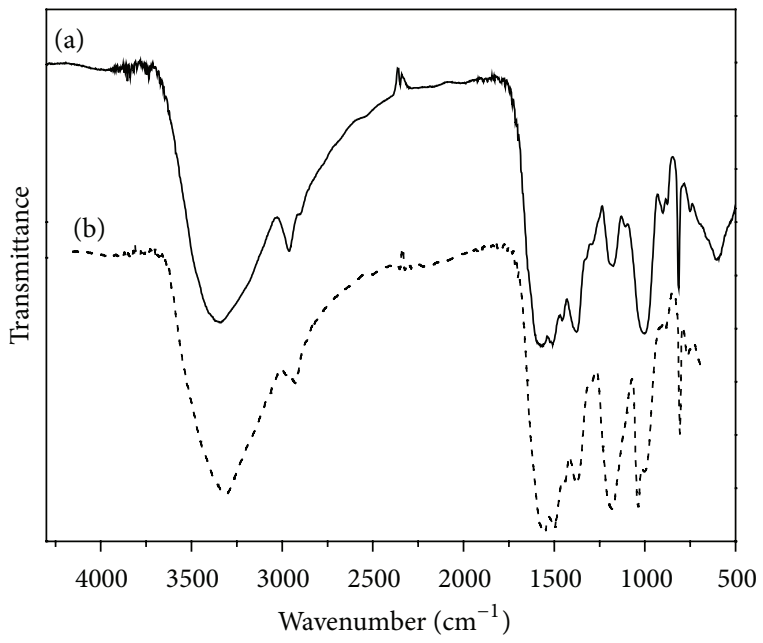

Figure 5: IR spectra of the SMF and NMS.

transporting, handling, and placing concrete. Table 2 shows the slump loss of concrete with NMS superplasticized at $1.0 \%$ dosage. Generally, the slump of superplasticized concrete by the use of SMF first slightly decreases with time and then decreases a bit quickly. The NMS superplasticized concrete maintains the initial slump values for about $60 \mathrm{~min}$, so the NMS resin has excellent capability of slump loss control and is more suitable for a preparation for pumping concrete. As can be seen, dispersing mechanism of water-reducing agent is not only related to molecular adsorption characteristics but also the configuration of the molecule which is shown in Figure 5. The molecular structure is based on the short linear branches, lying on the surface of cement particles and adsorbed thick layer [13]. Thus, dispersing ability based mainly from electrostatic repulsion force generating a double-electrical adsorption layer [14]. Therefore, the dispersing ability of NMS mainly comes from the electrostatic repulsive force produced by the adsorption double-electrical layer which resulted in that hydration products easily covered the molecules of NMS on the surface of cement particles.

3.6. Effect of NMS on Compressive and Flexural Strength. Results were shown in Figure 6 which showed that NMS increased concrete strength when compared to the control concrete. Thus, NMS increased compressive strength of concrete as the ratio of water/cementitious material decreased in the mixtures. This situation is similar for both curing

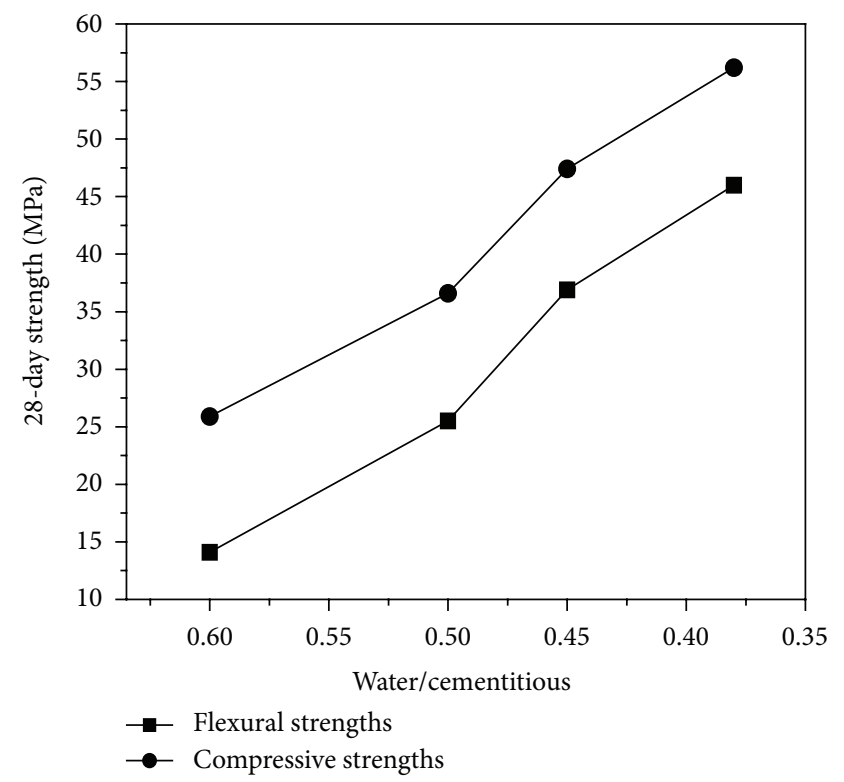

Figure 6: The compressive and flexural strength of superplasticized concrete at 28 days.

conditions. Most of the compressive strength of concrete produced was higher than that of the control concrete at all ages. The 28-day compressive strength values were 36.6, 47.4 , and $56.2 \mathrm{MPa}$ for $0.5,0.45$, and 0.38 water/cementitious ratios, respectively. Similar results were also obtained by Plank and Hirsch [15]. Flexural strengths of concrete by use of NMS in continuous moist curing were higher than those of concrete in open air curing. In general, as the ratio of water/cementitious increased flexural strengths of concrete increased when compared to control concrete as seen in Figure 6. Nevertheless, as the ratio of NMS increased, strength also increased when compared to control concrete for the 28-day strengths.

\section{Conclusions}

Water-soluble NMS resins were synthesized by the reaction among melamine, formaldehyde, and sulfonated glucose. The NMS resins synthesized could reduce the water content, improve the workability and compressive strength of concrete, and maintain the initial slump of concrete after $60 \mathrm{~min}$. The results indicate that the NMS resins are more effective in promoting the concrete performance of the 
workability, slump loss control, and compressive strength. Therefore, NMS has the potential to be used as a kind of high performance water-reducing admixture in concrete.

\section{Acknowledgment}

This project was supported by the Natural Science Foundation of Beijing Oriental YuHong Waterproof Technology Co. Ltd.

\section{References}

[1] P. J. Andersen, D. M. Roy, and J. M. Gaidis, "The effect of superplasticizer molecular weight on its adsorption on, and dispersion of, cement," Cement and Concrete Research, vol. 18, no. 6, pp. 980-986, 1988.

[2] M. Moukwa, D. Youn, and M. Hassanali, "Effects of degree of polymerization of water soluble polymers on concrete properties," Cement and Concrete Research, vol. 23, no. 1, pp. 122-130, 1993.

[3] M. Pei, D. Wang, X. Hu, and D. Xu, "Synthesis of sodium sulfanilate-phenol-formaldehyde condensate and its application as a superplasticizer in concrete," Cement and Concrete Research, vol. 30, no. 11, pp. 1841-1845, 2000.

[4] M. Pei, Y. Yang, X. Zhang, J. Zhang, and J. Dong, "Synthesis and the effects of water-soluble sulfonated acetone-formaldehyde resin on the properties of concrete," Cement and Concrete Research, vol. 34, no. 8, pp. 1417-1420, 2004.

[5] L. Yuan, G.-Z. Liang, J.-Q. Xie, and S.-B. He, "Synthesis and characterization of microencapsulated dicyclopentadiene with melamine-formaldehyde resins," Colloid and Polymer Science, vol. 285, no. 7, pp. 781-791, 2007.

[6] Y. Yuan, M. Rong, and M. Zhang, "Preparation and characterization of poly(melamine-formaldehyde) walled microcapsules containing epoxy," Acta Polymerica Sinica, no. 5, pp. 472-480, 2008.

[7] J. Hu, H.-Q. Chen, and Z. Zhang, "Mechanical properties of melamine formaldehyde microcapsules for self-healing materials," Materials Chemistry and Physics, vol. 118, no. 1, pp. 63-70, 2009.

[8] G. Sun and Z. Zhang, "Mechanical properties of melamineformaldehyde microcapsules," Journal of Microencapsulation, vol. 18, no. 5, pp. 593-602, 2001.

[9] S. Ahmad, S. M. Ashraf, E. Sharmin, M. Nazir, and M. Alam, "Studies on new polyetheramide-butylated melamine formaldehyde based anticorrosive coatings from a sustainable resource," Progress in Organic Coatings, vol. 52, no. 2, pp. 85-91, 2005.

[10] X.-M. Tong, T. Zhang, M.-Z. Yang, and Q. Zhang, "Preparation and characterization of novel melamine modified poly(ureaformaldehyde) self-repairing microcapsules," Colloids and Surfaces A, vol. 371, no. 1-3, pp. 91-97, 2010.

[11] M. Collepardi, "Admixtures used to enhance placing characteristics of concrete," Cement and Concrete Composites, vol. 20, no. 2-3, pp. 103-112, 1998.

[12] S. M. Lahallh, M. Absl-Halabi, and A. M. Ali, "Effect of polymerization conditions of sulfonated-melamine formaldehyde superplasticizers on concrete," Cement and Concrete Research, vol. 18 , no. 4, pp. 513-531, 1988.
[13] M. C. Vieira, D. Klemm, L. Einfeldt, and G. Albrecht, "Dispersing agents for cement based on modified polysaccharides," Cement and Concrete Research, vol. 35, no. 5, pp. 883-890, 2005.

[14] T. Zhang, S. Shang, F. Yin, A. Aishah, A. Salmiah, and T. L. Ooi, "Adsorptive behavior of surfactants on surface of Portland cement," Cement and Concrete Research, vol. 31, no. 7, pp. 10091015, 2001.

[15] J. Plank and C. Hirsch, "Impact of zeta potential of early cement hydration phases on superplasticizer adsorption," Cement and Concrete Research, vol. 37, no. 4, pp. 537-542, 2007. 

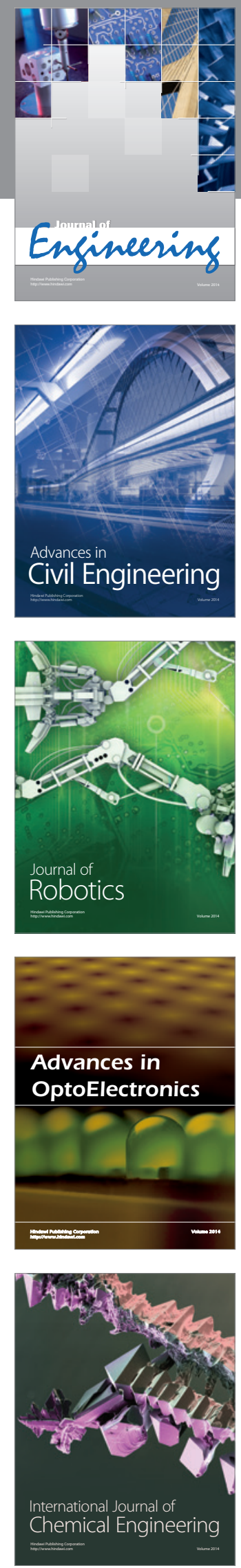

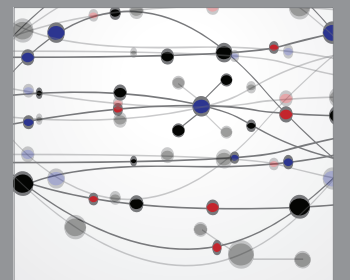

The Scientific World Journal
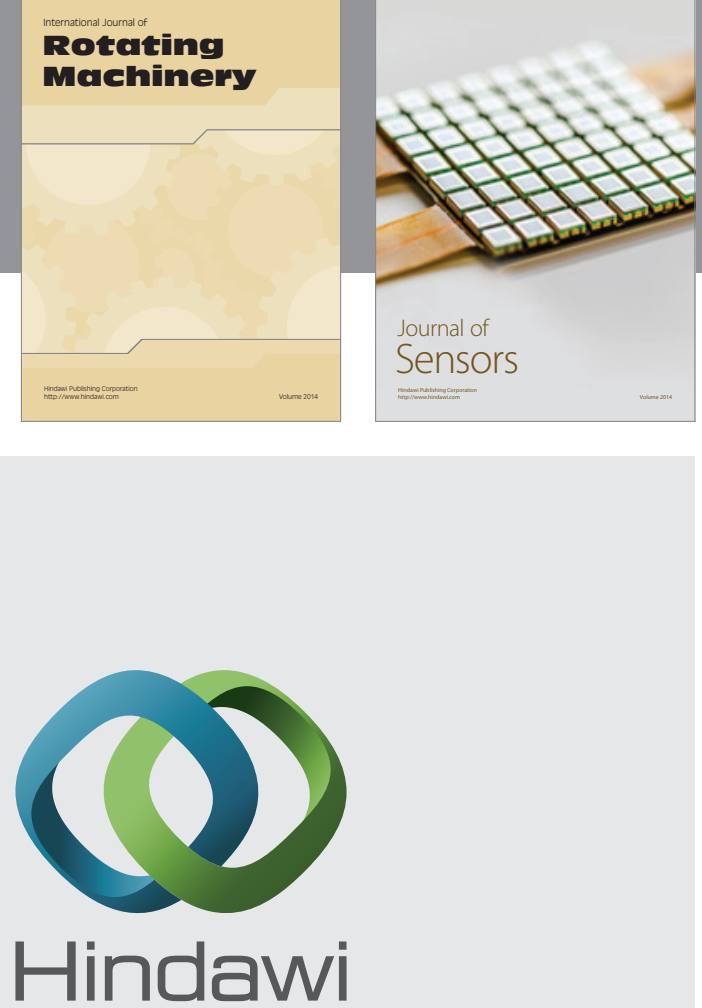

Submit your manuscripts at http://www.hindawi.com
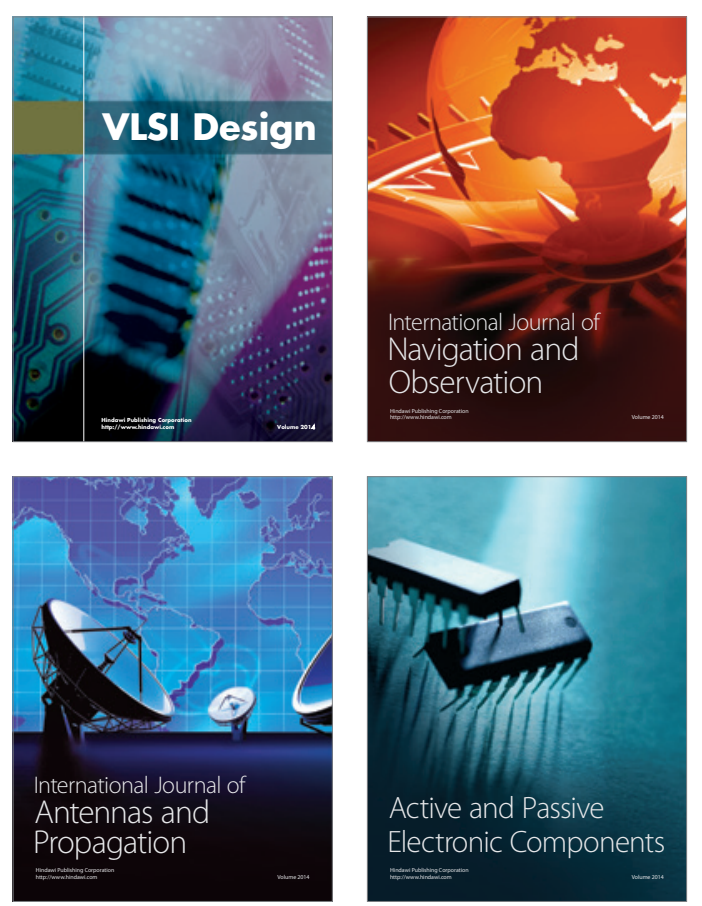
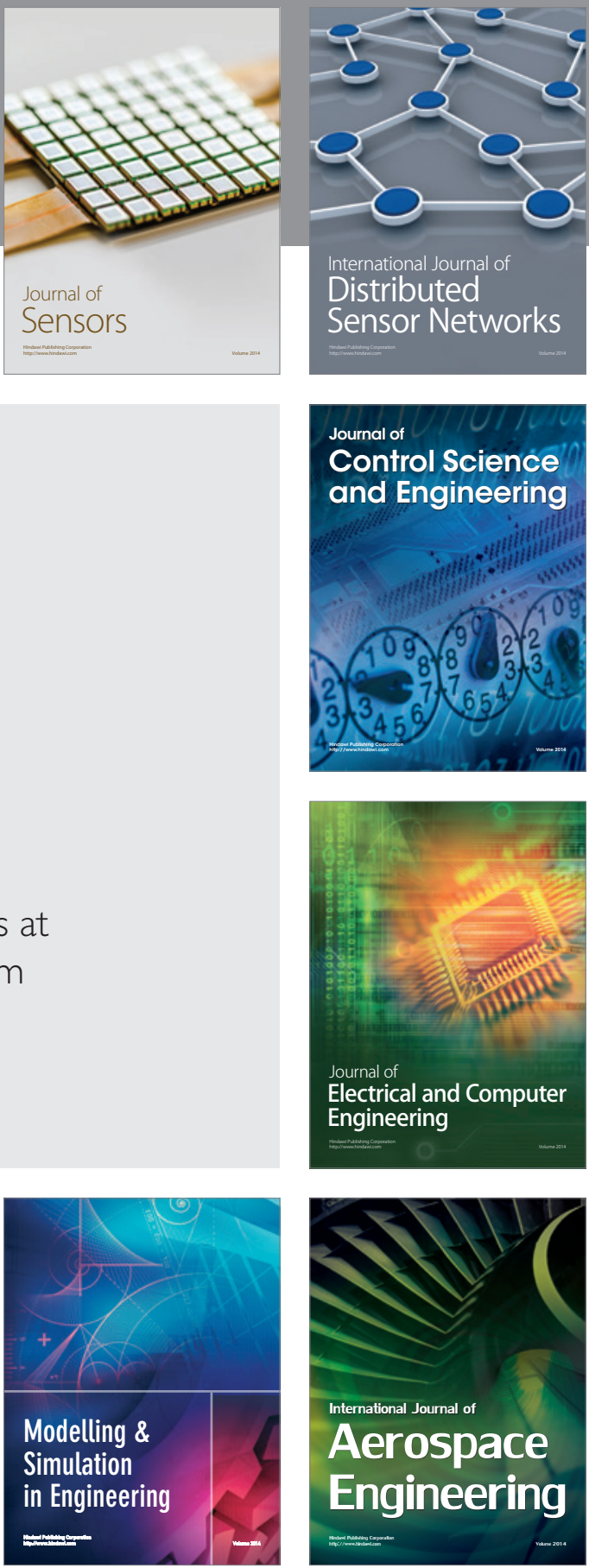

Journal of

Control Science

and Engineering
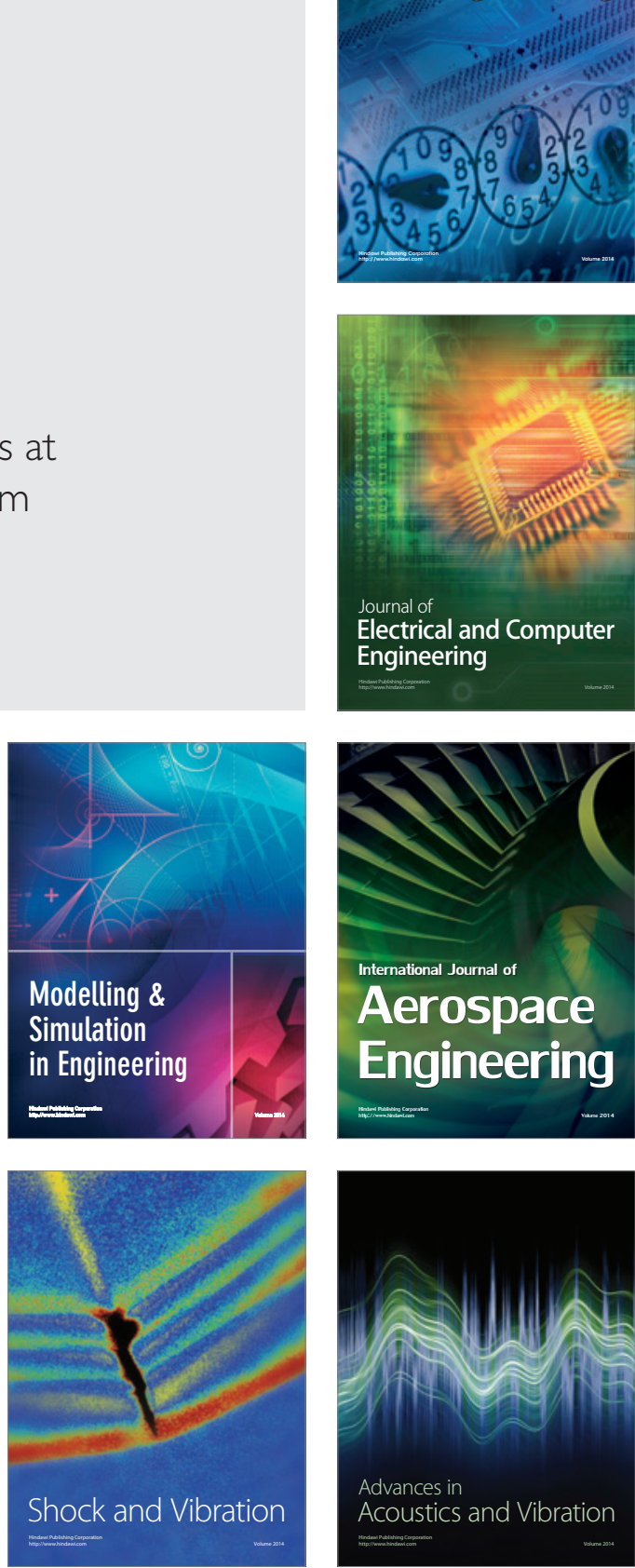